\title{
Tratamiento con brace termoplástico para fracturas de húmero
}

\author{
Agustina Dávalos, Eugenia Hernández, Paula Simaro, Elena Santamarina, Johanna Di Tullio, Martina Chiurazzi \\ Sector de Terapia Ocupacional, Hospital Italiano de Buenos Aires, Ciudad Autónoma de Buenos Aires, Argentina
}

\begin{abstract}
RESUMEN
Objetivo: Evaluar la satisfacción con el uso del brace termoplástico y el resultado funcional del tratamiento conservador en pacientes con fracturas de la diáfisis del húmero. Materiales y Métodos: Estudio retrospectivo de pacientes con fracturas de húmero cerradas, tratados con brace termoplástico hasta su consolidación y un seguimiento mínimo de 12 meses. Se registraron los siguientes datos: tipo de fractura y localización, mecanismo de lesión, miembro lesionado, tiempo de inmovilización con yeso y uso del brace, complicaciones y tiempo de consolidación. Se evaluaron el dolor mediante la escala analógica visual, la satisfacción con la escala de Likert, el balance articular con la escala de Constant y la funcionalidad según el puntaje QuickDASH. Resultados: Se incluyó a 17 pacientes (16 mujeres, 1 hombre; edad promedio 67 años). La inmovilización inicial con yeso fue de 13 días (rango 0-32). Los pacientes usaron el brace por 8.6 semanas (rango 3-16) hasta la consolidación radiográfica en la décima semana. El seguimiento promedio fue de 24 meses (rango 12-60) y el puntaje de dolor, de 0,5 (rango 1-3). El 59\% estuvo muy satisfecho con los resultados y el $41 \%$, satisfecho. El $59 \%$ logró una flexión del hombro $>150^{\circ}$, el $47 \%$, una abducción $>150^{\circ}$, el $41 \%$, una rotación interna con pulgar entre escápulas y el $47 \%$, una rotación externa de $70^{\circ}$. El puntaje QuickDASH promedio fue de 9. Conclusiones: Los pacientes se mostraron muy satisfechos con el uso del brace termoplástico para el tratamiento incruento de las fracturas de húmero y los resultados funcionales fueron aceptables.
\end{abstract}

Palabras clave: Húmero; fractura; brace termoplástico; tratamiento conservador.

Nivel de Evidencia: IV

\section{Thermoplastic Brace Treatment for Humerus Fractures}

\section{ABSTRACT}

Purpose: To evaluate the satisfaction with the use of a thermoplastic brace and the functional outcomes in the conservative treatment of patients with humeral shaft fractures. Materials and Methods: Retrospective study of patients with closed humerus fractures, treated with a thermoplastic brace until union and with a minimum follow-up of 12 months. We recorded the type and location of the fracture, mechanism of injury, injured limb, time of immobilization with plaster and use of brace, complications, and time of consolidation. The evaluation was performed using the visual analog scale (VAS) for pain, the Likert scale for patient satisfaction, the Constant scale for joint balance, and the QuickDash score for functionality. Results: 17 patients were included (16 female, 1 male), with an average age of 67 years. The initial plaster immobilization lasted 13 days (range 0-32). The patients wore the brace for 8.6 weeks (range 3-16) until radiographic consolidation in the $10^{\text {th }}$ week. The average follow-up was 24 months (range 12-60) and the pain score was 0.5 (range 1-3). 59\% were very satisfied with the results and $41 \%$ were satisfied. $59 \%$ achieved a shoulder flexion $>150^{\circ} ; 47 \%$, an abduction $>150^{\circ} ; 41 \%$, an internal rotation with thumb between scapulae; and $47 \%$, an external rotation of $70^{\circ}$. The average QuickDASH score was 9. Conclusion: The use of a thermoplastic brace in the conservative treatment of humerus fractures presented high patient satisfaction and acceptable functional outcomes for the affected limb.

Keywords: Humerus; fractures; thermoplastic brace; conservative treatment.

Level of Evidence: IV

\section{INTRODUCCIÓN}

El manejo conservador de las fracturas de la diáfisis humeral es una alternativa de común elección, con aceptables resultados publicados y una tasa de falta de consolidación que oscila entre el $1 \%$ y el $12 \%$, según distintos reportes. ${ }^{1}$

\footnotetext{
Recibido el 20-4-2021. Aceptado luego de la evaluación el 11-5-2021 • Lic. T. O. AGUSTINA DAVALOS • toagustinadavalos@gmail.com Cómo citar este artículo: Dávalos A, Hernández E, Simaro P, Santamarina E, Di Tullio J, Chiurazzi M. Tratamiento con brace termoplástico para fracturas de húmero. Rev Asoc Argent Ortop Traumatol 2021;86(5):575-580. https://doi.org/10.15417/issn.1852-7434.2021.86.5.1353.
} 
Actualmente, el brace de húmero es considerado el tratamiento conservador estándar para las fracturas no quirúrgicas de húmero. Esta técnica fue popularizada por Sarmiento, quien describió y desarrolló una alternativa terapéutica para lograr la consolidación ósea mientras se mantiene la movilidad articular, reduciendo así los tiempos y costos de rehabilitación, y las complicaciones quirúrgicas. ${ }^{2}$

El fundamento del tratamiento con brace consiste en proveer alineación y estabilidad a la fractura (limitando el movimiento en el foco de fractura), comprimiendo los músculos y tejidos blandos circundantes al hueso lesionado, permitiendo un movimiento controlado de las articulaciones proximales y distales a la fractura, estimulando así la consolidación ósea. Las fuerzas ejercidas durante la contracción muscular se traducen en fuerzas de compresión y coaptación que estabilizan mecánicamente la fractura. Es decir, la compresión no depende de la fuerza del material, sino del tamaño y la forma del brace termoplástico que permite ejercer una presión constante sobre la fractura durante la contracción muscular activa. La contracción de la musculatura que transcurre paralela al eje del húmero (bíceps, tríceps y braquial anterior) permite restablecer la alineación y la rotación adecuadas, lo cual explicaría por qué las deformidades funcionales son raras si hay movimiento activo. ${ }^{3}$

Las contraindicaciones formales del brace incluyen: fracturas asociadas a defectos de tejidos blandos, compromiso vascular o neurológico, excesiva angulación o acortamiento humeral y escaso compromiso del paciente para cumplir con este tipo de tratamiento. ${ }^{4}$

El objetivo de este estudio fue evaluar la satisfacción con el uso del brace termoplástico y el resultado funcional del tratamiento conservador de pacientes con fracturas de la diáfisis del húmero.

\section{MATERIALES Y MÉTODOS}

Se realizó un estudio retrospectivo entre junio de 2013 y junio de 2018. Los criterios de inclusión fueron: pacientes de ambos sexos con fractura de húmero cerrada, tratados con brace termoplástico confeccionado por el mismo equipo de terapistas, con cumplimiento efectivo del equipamiento hasta su consolidación y un seguimiento mínimo de 12 meses. Los criterios de exclusión fueron: pacientes con lesiones asociadas, no cumplir con el uso del brace hasta la consolidación y seguimiento inadecuado.

Los pacientes fueron inmovilizados inicialmente con un yeso y luego fueron derivados al Servicio de Terapia Ocupacional, donde se confeccionó el brace termoplástico de baja temperatura.

El brace consiste en dos valvas confeccionadas a medida con material termoplástico de 2,4 $\mathrm{mm}$ de espesor, ajustado con dos piezas de velcro de $3 \mathrm{~cm}$ de ancho (Figura 1).
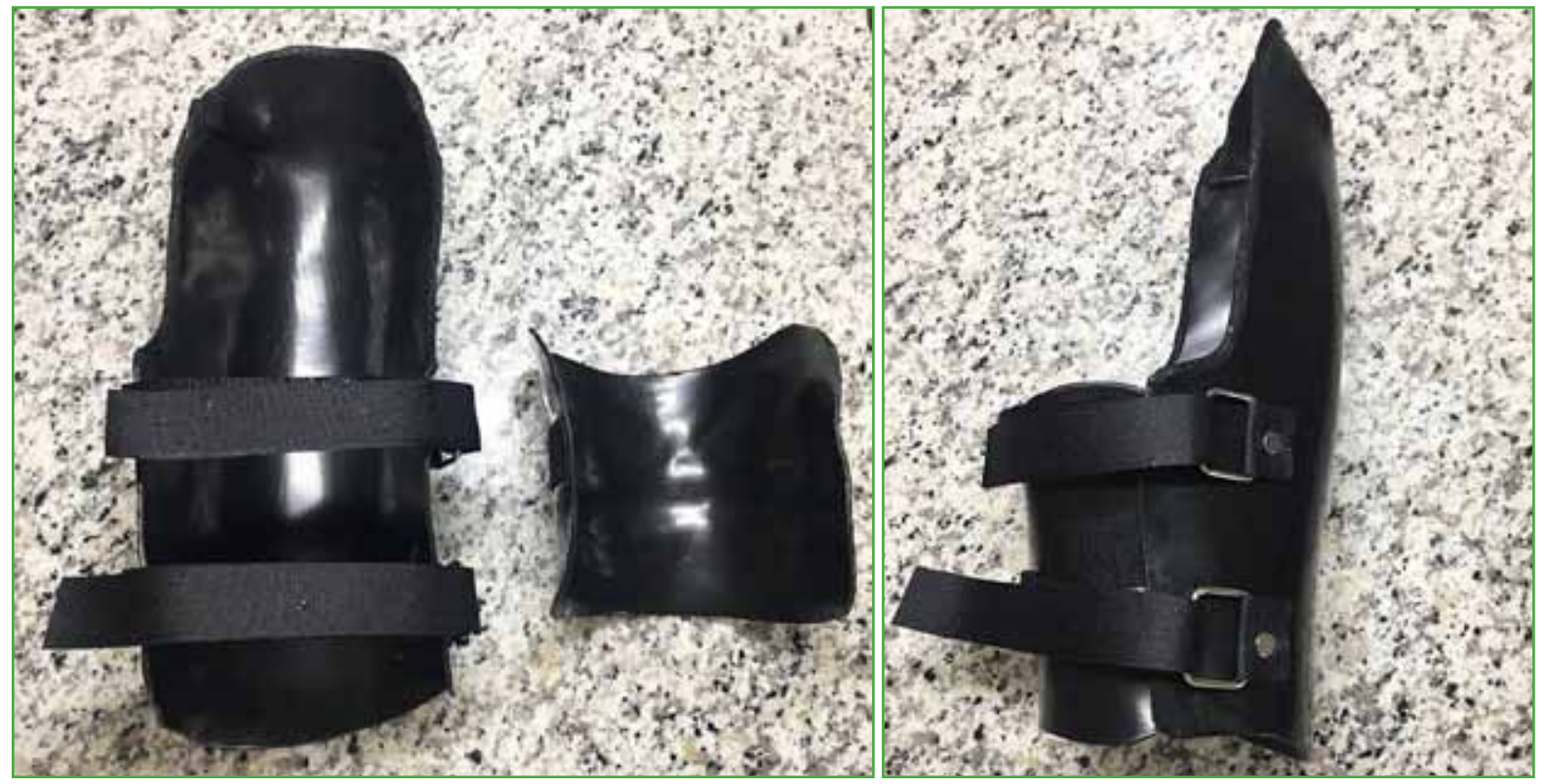

Figura 1. Brace termoplástico utilizado en el tratamiento de fracturas de húmero, con sus valvas medial y lateral conformadas y separadas. 


\section{Procedimiento de confección del brace}

1. El brace se moldeó sobre cada paciente, de forma anatómica. La valva interna se moldeó sobre la cara interna del húmero, $2 \mathrm{~cm}$ por debajo de la axila y se la sujetó con velcro; la valva externa se moldeó sobre la cara lateral del brazo, extendiéndose inmediatamente debajo del acromion, dejando libre la articulación del codo.

2. Sobre los laterales de la valva externa se colocaron herrajes (a $1 \mathrm{~cm}$ del borde delantero del brace) con el objetivo de poder ajustar los velcros de manera suficiente para que el equipamiento realice la coaptación adecuada (Figura 2).

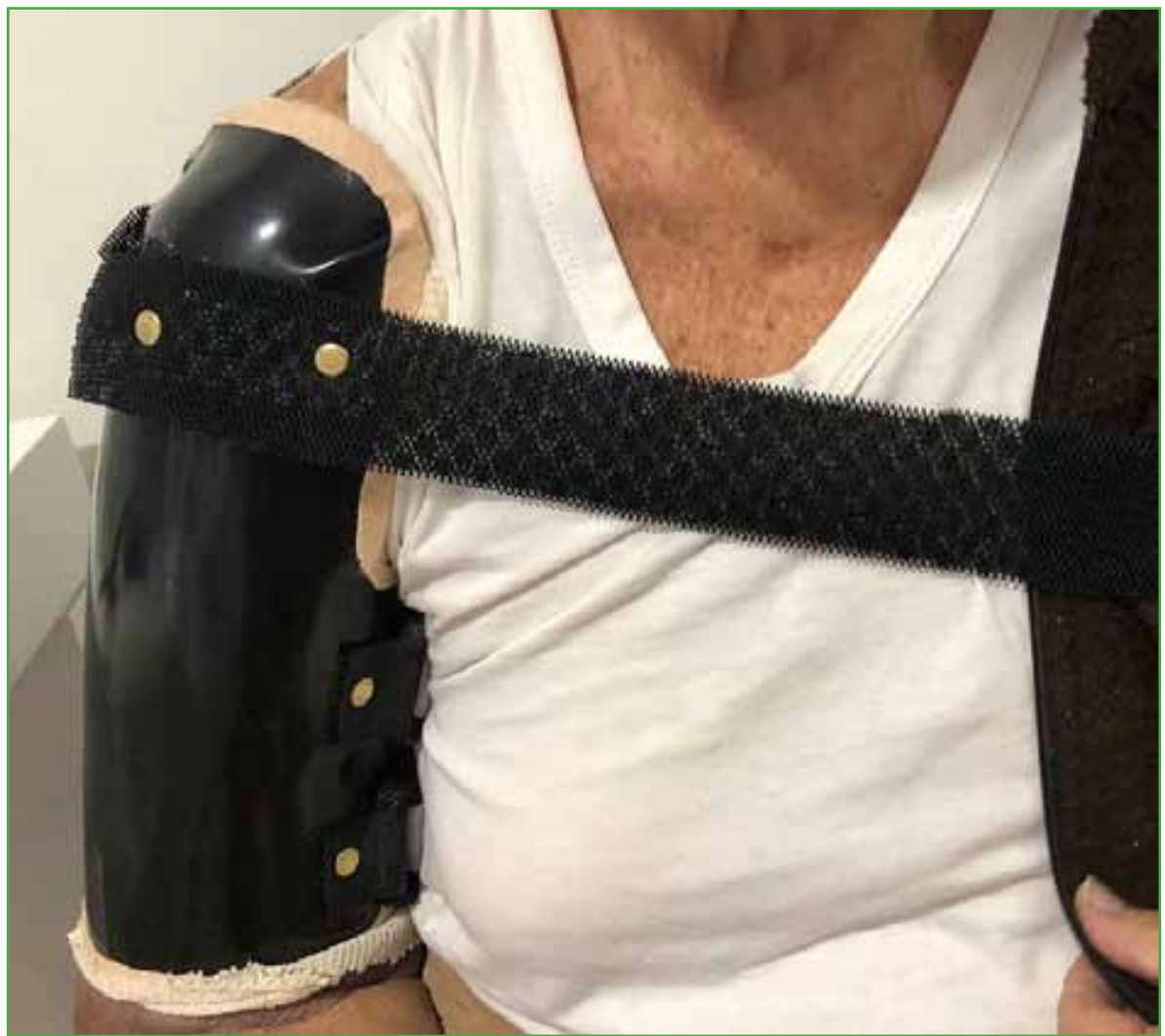

Figura 2. Brace termoplástico colocado. Sobre los laterales de la valva externa se colocaron herrajes para poder ajustar los velcros y lograr la coaptación adecuada.

3. Sobre el extremo proximal de la ortesis, a la altura de la cara posterior del hombro, se colocó un velcro rodeando el contorno del cuerpo, pasando debajo de la axila del miembro contralateral, adherido finalmente a la ortesis a la altura de la cara anterior del hombro.

4. Los bordes de la ortesis fueron cubiertos con felpa o goma eva. Se colocó una malla tubular (tres capas de 1,5 $\mathrm{mm}$ de espesor) entre la piel y la ortesis para prevenir la irritabilidad y las lesiones de la piel.

5. Se indicaron el uso de cabestrillo y ejercicios activos de articulaciones libres.

Durante el primer mes de uso del brace, se controló a los pacientes semanalmente, evaluando el confort con el uso, la presencia de dolor, el estado de la piel y la higiene de la extremidad. Si el equipamiento se encontraba en adecuadas condiciones, sin evidencia de lesiones cutáneas asociadas y el paciente se sentía cómodo y cumplía con el tratamiento, no se lo volvió a citar hasta el momento de la consolidación. Luego se solicitó una radiografía con el brace colocado que fue controlada, en todos los casos, por el equipo médico, y se repitió al mes, a los dos meses y a los tres meses de la fractura. Se instruyó al paciente a mantener la extremidad dentro del cabestrillo y a quitárselo para realizar la rutina de ejercicios de flexo-extensión de codo, movilidad de muñeca y dedos (3 veces por día, 20 repeticiones). Se brindaron también pautas de cuidado para el control y el tratamiento del edema. 
La indicación de distanciar el uso del cabestrillo y del brace estuvo en manos del equipo médico tratante, en función de la evidencia clínica y radiográfica de consolidación.

Se recolectaron datos de la historia clínica sobre el sexo, la edad en el momento de la fractura, el mecanismo de lesión, el miembro superior lesionado, el tiempo de inmovilización con yeso (días), el tiempo de uso del brace (semanas), el tiempo de consolidación (semanas) y las complicaciones durante el uso del brace.

Se realizó un seguimiento a largo plazo después de la consolidación. Se contactó a los pacientes por vía telefónica y se los citó para una evaluación. La valoración subjetiva se realizó mediante la escala analógica visual para dolor; la satisfacción con el uso del brace se determinó con la escala de Likert de 4 ítems $(1=$ insatisfecho, $2=$ poco satisfecho, 3 = satisfecho, 4 = muy satisfecho); la movilidad del hombro, con el apartado de balance articular de la escala de Constant y la funcionalidad, con el puntaje QuickDASH. Se registraron como observaciones aquellos datos referidos por el paciente en relación con el equipamiento.

Se utilizó el programa Stata para la evaluación estadística.

\section{RESULTADOS}

Diecisiete de 31 pacientes tratados con un brace por fracturas de húmero cumplieron con los criterios de inclusión. El 53\% de las fracturas se asentaban en la unión del tercio medio y proximal (9/17); el 35\%, en el tercio medio (6/17) y el 6\%, en la unión del tercio medio y distal (1/17). Según la clasificación AO, seis fracturas eran tipo A1; una, A2; una, A3; cinco, B1; una, B2 y tres, C1. La edad promedio al sufrir la lesión fue 67 años (rango 30-90). La muestra estaba formada por 16 mujeres (94\%) y un hombre. Diez pacientes tenían compromiso del húmero derecho y siete, del izquierdo. Todas las fracturas eran cerradas. En todos los pacientes, el mecanismo de la lesión fue de baja energía por caída desde su propia altura.

El tiempo de inmovilización con yeso, antes de colocar el brace fue, en promedio, de 13 días (rango 0-32). El tiempo promedio de uso del brace termoplástico fue de 8.6 semanas (rango 3-16). Todos utilizaron el brace hasta el momento de la consolidación radiográfica de la fractura, que ocurrió, en promedio, a las 10 semanas (rango 6-16). El tiempo promedio de seguimiento fue de 24 meses (rango 12-60).

El puntaje promedio de dolor en la escala analógica visual fue de 0,5 (rango 1-3).

En cuanto a la satisfacción de los pacientes con el uso del brace, según la escala de Likert, el 59\% (10/17) manifestó estar muy satisfecho y el 41\% (7/17), estar satisfecho (7/17). Ninguno refirió insatisfacción en esta serie.

Las complicaciones durante el uso del brace fueron: edema en la mano y molestias en la piel, todas transitorias. Ningún paciente sufrió una lesión tisular.

Las observaciones por parte de los pacientes incluyeron: sugerencia de incorporar una malla tubular más gruesa; otros resaltaron la importancia y la necesidad del control y la higiene, la evaluación de herrajes y sujeciones, porque generaban molestias y molestia en los bordes de la ortesis.

El 47\% (8/17) de los pacientes recibió indicación de rehabilitación (kinesiología o Terapia Ocupacional).

Respecto a la movilidad del hombro (apartado de balance articular de la escala de Constant), el 59\% (10/17) logró una movilidad de flexión del hombro $>150^{\circ}$ y el $47 \%$ (8/17), una abducción del hombro $>150^{\circ}$. En cuanto a la rotación interna del hombro, el $41 \%$ (7/17) pudo llevar el pulgar entre las escápulas y en rotación externa, el $47 \%$ (8/17) alcanzó, en promedio, los $70^{\circ}$ en la medición goniométrica.

La funcionalidad (QuickDASH) fue, en promedio, de 9 (rango 0-48; desviación estándar 15,3; mediana 2,3).

Dado que, a mayor puntaje, mayor discapacidad, sobre la base de los resultados (QuickDASH promedio 9), cabe señalar que se lograron resultados funcionales excelentes/buenos (Tabla).

\section{DISCUSIÓN}

Según la bibliografía, se recomienda el tratamiento conservador con el uso del brace para las fracturas diafisarias de húmero, pues ofrece una alta tasa de consolidación, buenos resultados funcionales y comodidad para el paciente. ${ }^{1-4}$

El brace, por lo general, se utiliza en el tratamiento de las fracturas cerradas ocasionadas por mecanismos de baja energía, que requieren poca o nula reducción, la cual se produce, de manera espontánea, por el efecto de la gravedad y la consiguiente estabilización. .,6 $^{5}$

La experiencia clínica de algunos autores ha mostrado que las fracturas humerales tratadas con brace tienen una alta tasa de consolidación y que la recuperación de la función es excelente, salvo cuando ocurren complicaciones neurovasculares y de partes blandas (especialmente con una lesión concomitante del nervio radial). Los buenos resultados obtenidos en este estudio refuerzan la consideración del brace como una herramienta útil en el tratamiento conservador de las fracturas de húmero. ${ }^{9}$ 
Tabla. Resumen de la serie de pacientes con fracturas de húmero tratados con brace termoplástico

\begin{tabular}{|c|c|c|c|c|c|c|c|c|c|}
\hline Caso & Sexo & Edad & Lado & $\begin{array}{l}\text { Tiempo } \\
\text { de uso } \\
\text { del brace } \\
\text { (semanas) }\end{array}$ & $\begin{array}{c}\text { Tiempo hasta } \\
\text { la } \\
\text { consolidación } \\
\text { (semanas) }\end{array}$ & $\begin{array}{c}\text { Seguimiento } \\
\text { (meses) }\end{array}$ & $\begin{array}{l}\text { Dolor } \\
\text { (EAV) }\end{array}$ & QuickDASH & Satisfacción \\
\hline 1 & $\mathrm{~F}$ & 88 & MSD & 3 & 6 & 29 & $0 / 10$ & 0 & Satisfecho \\
\hline 2 & $\mathrm{~F}$ & 52 & MSI & 6 & 8 & 28 & $0 / 10$ & 2.3 & Muy satisfecho \\
\hline 3 & $\mathrm{~F}$ & 67 & MSD & 6 & 7 & 12 & $0 / 10$ & 2.3 & Muy satisfecho \\
\hline 4 & F & 75 & MSI & 8 & 8 & 44 & $0 / 10$ & 6.8 & Satisfecho \\
\hline 5 & $\mathrm{~F}$ & 30 & MSI & 8 & 12 & 13 & $3 / 10$ & 6.8 & Satisfecho \\
\hline 6 & $\mathrm{~F}$ & 69 & MSD & 9 & 13 & 32 & $0 / 10$ & 2.3 & Muy satisfecho \\
\hline 7 & $\mathrm{~F}$ & 52 & MSD & 6 & 8 & 55 & $0 / 10$ & 2.3 & Muy satisfecho \\
\hline 8 & M & 65 & MSD & 16 & 16 & 12 & $0 / 10$ & 2.3 & Muy satisfecho \\
\hline 9 & $\mathrm{~F}$ & 61 & MSI & 7 & 11 & 60.5 & $1 / 10$ & 6.8 & Muy satisfecho \\
\hline 10 & $\mathrm{~F}$ & 64 & MSD & 12 & 12 & 12 & $0 / 10$ & 4.5 & Muy satisfecho \\
\hline 11 & $\mathrm{~F}$ & 53 & MSD & 16 & 16 & 25 & $0 / 10$ & 0 & Muy satisfecho \\
\hline 12 & $\mathrm{~F}$ & 83 & MSI & 10 & 10 & 21 & $0 / 10$ & 0 & Satisfecho \\
\hline 13 & $\mathrm{~F}$ & 90 & MSD & 7.5 & 7.5 & 12 & $0 / 10$ & 1.8 & Satisfecho \\
\hline 14 & F & 86 & MSD & 8 & 8 & 12 & $2 / 10$ & 4.8 & Satisfecho \\
\hline 15 & $\mathrm{~F}$ & 71 & MSI & 6 & 8 & 12 & $3 / 10$ & 4.8 & Satisfecho \\
\hline 16 & F & 81 & MSI & 8 & 11 & 12 & $0 / 10$ & 0 & Muy satisfecho \\
\hline 17 & $\mathrm{~F}$ & 59 & MSD & 9.5 & 11 & 20 & $0 / 10$ & 0 & Muy satisfecho \\
\hline
\end{tabular}

$\mathrm{M}=$ masculino, $\mathrm{F}$ = femenino, $\mathrm{MSD}=$ miembro superior derecho, $\mathrm{MSI}=$ miembro superior izquierdo, EAV = escala analógica visual .

El incumplimiento del tratamiento se menciona, en ocasiones, como una contraindicación para el brace. En la serie de casos tratados, el cumplimiento del uso del brace fue bueno y la tasa de satisfacción con el equipamiento fue alta.

En la bibliografía, hay consenso en que se deben realizar ciertos ejercicios y en rangos protegidos de articulaciones libres y que se deben evitar los movimientos de flexión y abducción del hombro, al igual que el apoyo con el brazo. ${ }^{6-8,10,11}$

Cabe mencionar que múltiples estudios evaluaron los resultados funcionales después del tratamiento con el brace e informaron pérdidas mínimas del rango de movilidad, que no provocaron limitaciones en las actividades cotidianas. En nuestro estudio, se obtuvieron resultados similares. , $^{4,8}$

En cuanto a las complicaciones generales y específicas de las fracturas de húmero, se asocian, con frecuencia, al tratamiento quirúrgico. ${ }^{8}$ En la serie de casos estudiados, no hubo complicaciones significativas secundarias al tratamiento. A menudo, se mencionan los problemas dermatológicos por el tratamiento conservador; en nuestro estudio, no se registraron dichos problemas, no se constataron lesiones de la piel por el uso del brace. El adecuado control de la ortesis y la higiene de la piel pueden reducir, al mínimo, el riesgo de dicha complicación. ${ }^{10-12}$

Este estudio presenta ciertas limitaciones: se trata de una serie retrospectiva de una pequeña muestra. A su vez, existe heterogeneidad en cuanto al tipo de fracturas de húmero incluidas en el estudio. Sin embargo, mencionamos como fortalezas el establecimiento de un protocolo común y detallado de ortesis por un mismo grupo de terapistas, el seguimiento por un mismo servicio médico hasta la consolidación y el seguimiento mínimo considerado acorde para este cuadro. Consideramos que los estudios prospectivos pueden colaborar con una evaluación más rigurosa y aportar un mayor nivel de evidencia. 


\section{CONCLUSIONES}

Los resultados de nuestro estudio sugieren que el uso de un brace termoplástico con una correcta coaptación y supervisión del profesional permite lograr la consolidación ósea en las fracturas diafisarias de húmero. El control del dolor junto con la satisfacción del paciente por el confort del brace valida a esta opción terapéutica para esta enfermedad. Es fundamental su confección adecuada para mejorar la tolerancia por parte del paciente, ya que el uso debe ser prolongado, y se debe considerar en pacientes seleccionados; además, permite disminuir los costos de quirófano y los riesgos de infección por la cirugía.

Conflicto de intereses: Los autores no declaran conflictos de intereses.

ORCID de E. Hernández: https://orcid.org/0000-0003-1973-2001 ORCID de P. Simaro: https://orcid.org/0000-0001-6122-2326 ORCID de E. Santamarina: https://orcid.org/0000-0001-5344-6565
ORCID de J. Di Tullio: https://orcid.org/0000-0001-6413-0160 ORCID de M. Chiurazzi: https://orcid.org/0000-0002-9642-6836

\section{BIBLIOGRAFÍA}

1. Sarmiento A, Kinman PB, Galvin EG, Schmidtt RH, Phillips JG. Functional bracing of fractures of the shaft of the humerus. J Bone Joint Surg Am 1977;59(5):596-601. PMID:873955

2. Updegrove GF, Mourad W, Abboud J. Humeral shaft fractures. J Shoulder Elbow Surg 2018;27(4):e87-e97. https://doi.org/10.1016/j.jse.2017.10.028

3. Colditz JC. Functional fracture bracing. En: Skirven T, Osterman A, Fedorczyk J, Amadio P. Rehabilitation of the hand and upper extremity, $6^{\text {th }}$ ed., Philadelphia: Elsevier Mosby; 2011:1620-9.

4. Naver L, Aalberg R. Humeral shaft fractures treated with a ready-made fracture brace. Arch Orthop Trauma Surg 1986;106(1):20-2. https://doi.org/10.1007/BF00435647

5. Walker M, Palumbo B, Badman B, Brooks J, Van Gelderen J, Mighell M. Humeral shaft fractures: a review. J Shoulder Elbow Surg 2011;20(5):833-44. https://doi.org/10.1016/j.jse.2010.11.030

6. Pehlivan O. Functional treatment of the distal third humeral shaft fractures. Arch Orthop Trauma Surg 2002; 122(7):390-95. https://doi.org/10.1007/s00402-002-0403-x

7. Ali E, Griffiths D, Obi N, Tytherleigh-Strong G, Van Rensburg L. Nonoperative treatment of humeral shaft fractures revisited. J Shoulder Elbow Surg. 2015;24(2):210-4. https://doi.org/10.1016/j.jse.2014.05.009

8. Rutgers M, Ring D. Treatment of diaphyseal fractures of the humerus using a functional brace. J Orthop Trauma 2006;20(9):597-601. https://doi.org/10.1097/01.bot.0000249423.48074082

9. Sarmiento A, Zagorski JB, Zych GA, Latta LL, Capps CC. Functional bracing for the treatment of fractures of the humeral diaphysis. J Bone Joint Surg Am 2000;82(4):478-86. https://doi.org/10.2106/00004623-200004000-00003

10. Wallny T, Sagebiel C, Westerman K, Wagner UA, Reimer M. Comparative results of bracing and interlocking nailing in the treatment of humeral shaft fractures. Int Orthop 1997;21(6):374-9. https://doi.org/10.1007/s002640050189

11. Zagorski JB, Latta LL, Zych GA, Finnieston AR. Diaphyseal fractures of the humerus. Treatment with prefabricated braces. J Bone Joint Surg Am 1988;70(4):607-10. PMID: 3356728

12. Koch PP, Gross DF, Gerber C. The results of functional (Sarmiento) bracing of humeral shaft fractures. J Shoulder Elbow Surg 2002;11(2):143-50. https://doi.org/10.1067/mse.2002.121634 